\title{
Development of an EliSPOT assay for detection of CMV-specific immune response
}

\author{
Maria Elena Terlizzi, Sara Astegiano, Francesca Sidoti, Stefano Gambarino, Rossana Cavallo, Cristina Costa, \\ Massimiliano Bergallo \\ SCDU Virology, AOU San Giovanni Battista, Torino, Italy
}

Key words: EliSPOT assay, CMV, immune response

Messa a punto di un saggio EliSPOT per la rilevazione della risposta immunitaria CMV-specifica

\section{SUMMARY}

Introduction: The Cytomegalovirus (CMV) is the major cause of morbidity and mortality in solid organ (SOT) and bone marrow (BMT) transplantation. An early reconstitution of immune response is crucial in limiting CMV replication; on the other hand, a late reconstitution may determine CMV reactivation with possible evolution to symptomatic phase. The Enzyme-Linked Immunosorbent Spot (EliSPOT) assay is a useful tool for monitoring the CMV-specific immune response recovery in transplant patients. This study propose the development and optimization of a interferon-gamma-(IFN- $\gamma$ )-based EliSPOT assay for the detection of CMV immune response.

Methods: CD3 + lymphocytes were separated using Robosep (negative selection, HLA ® EasySep WB Human T Cell Enrichment Kit, Stemcell Technology, Vancouver, Canada). The EliSPOT assay was optimized using the Human IFN gamma ELISPOT Kit and a modified protocol provided by Nanogen Advanced Diagnostics (Buttigliera Alta, Italy). Different parameters were analyzed: number of cells (200,000 300,000 ), antigens (CMV peptide mix and whole CMV antigen), incubation time (18 - 20 - 22 - 24h), number of washes, incubation conditions with secondary antibody and conditions of substrate development.

Results: Herein we report the optimal conditions identified. 200,000 CD3+ cells per well were stimulated with CMV peptide mix antigens. Cells were stimulated for $18 \mathrm{~h}$ at $37{ }^{\circ} \mathrm{C}$ in humidified atmosphere; wells were washed and secondary antibody was added and incubated at room temperature for $2 \mathrm{~h}$. After incubation a second series of washes were performed. Substrate was added and incubation for 15 minutes was carried out. The reaction was detected by plate reader AID ELISPOT (Strassberg, Germany).

Conclusions: We developed an EliSPOT assay for the detection of CMV-immune response and reconstitution. All variables were compared to previous published protocols. The improvement of the EliSPOT assay for the detection of CMV-specific immune response represents the first step for result evaluation in clinical practice.

\section{INTRODUZIONE}

L'utilizzo di regimi immunosoppressivi antirigetto ha portato all'emergenza di gravi patologie da infezione primaria o da riattivazione di virus latenti. Tra questi, il CMV rappresenta la principale causa di morbilità e mortalità nei trapiantati di organo solido (SOT) o midollo (BMT) (1). Nel paziente immunodepresso, una precoce ricostituzione dell'immunità cellulare gioca un ruolo fondamentale nel controllo della replicazione citomegalica e dell'infezione; per contro una ricostruzione tardiva può condurre all'insorgenza di infezioni ricorrenti che raggiungono la fase sintomatica se non opportunatamente trattate (3). In questo contesto assume fondamentale importanza il monitoraggio immunologico, associato a quello virologico, per discriminare tra pazienti responders (early - late) e non responders. Il saggio EliSPOT si propone come strumento efficace nel monitoraggio della ricostituzione immunologica CMV-specifica dei pazienti trapiantati. La metodica consente di analizzare la risposta (interferone-gamma mediata) del comparto T linfocitario nei confronti del virus, mediante l'utilizzo di antigeni CMV-specifici (2). In questo studio è stato messo a punto e ottimizzato un saggio EliSPOT per la rilevazione della risposta interferonmediata anti-CMV.

\section{MATERIALI E METODI}

I linfociti CD3+ sono stati separati negativamente utilizzando un separatore automatico, Robosep (EasySep ${ }^{\circledR}$ HLA WB Human T Cell Enrichment Kit, StemCell Technology). Il saggio EliSPOT è stato ottimizzato modificando un protocollo fornito dalla ditta Nanogen, utilizzando il kit Elispot Interferon- $\gamma$ Basis Kit. Sono stati analizzati diversi parametri: il numero di cellule da stimolare (200.000 - 300.000); tipo di antigene stimolante (CMV Peptide mix, CMV Fully antigen); tempo di incubazione (18 - 20 - 22 - 24h); tempo e numero di lavaggi; condizioni di incubazione con l'anticorpo secondario e condizioni di sviluppo del substrato.

\section{RISULTATI}

Le condizioni ideali prevedono un numero di linfociti CD3+ pari a 200.000/pozzetto, stimolati con l'antigene CMV Peptide mix, risultato maggiormente immunogeno. La stimolazione di $18 \mathrm{~h}$ a $37^{\circ} \mathrm{C}$ in camera umida ha preceduto una serie di lavaggi e l'incubazione con l'anticorpo secondario a temperatura ambiente in camera umida. Ad una seconda serie di lavaggi è seguita l'incubazione con il substrato per 15 minuti. L'avvenuta reazione è stata letta mediante il lettore di piastre AID.

\section{CONCLUSIONI}

In questo studio è stato messo a punto e ottimizzato un saggio EliSPOT per la rilevazione della ricostituzione della risposta anti-citomegalica. Sono state confrontate tutte le variabili operative derivanti dalle informazioni del fabbricante e da protocolli della letteratura. L'ottimizzazione della metodica è il primo passo per la valutazione clinica dei risultati.

\section{Ringraziamenti.}

Questo studio è stato finanziato dalla Fondazione ISI e Fondazione CRT nell'ambito del Progetto Lagrange.

\section{BIBLIOGRAFIA}

1. Chiereghin A, Gabrielli L, Zanfi C, et al. Monitoring cytomegalovirus Tcell immunity in small bowel/multivisceral transplant recipients. Transplant Proc. 2010 Jan-Feb; 42(1): 69-73.

2. Maecker HT, Hassler J, Payne JK, et al. Precision and linearity targets for validation of an IFNgamma ELISPOT, cytokine flow cytometry, and tetramer assay using CMV peptides. BMC Immunol. 2008 Mar 17; 9: 9.

3. Pourgheysari B, Piper KP, McLarnon A, et al. Early reconstitution of effector memory CD4+ CMV-specific T cells protects against CMV reactivation following allogeneic SCT. Bone Marrow Transplant. 2009 Jun; 43(11): 853-61. Epub 2008 Dec 22.

\section{Corresponding author: Cristina Costa}

I0126 Torino, Italy - Via Santena 9

Phone: 0039(I I)6705630 - Fax: 0039(I I)6705648

E-mail: cristina.costa@unito.it 